Proceedings of the 2008 Numerical Relativity Data Analysis Meeting, Syracuse University, Syracuse, NY, USA, 11-14 August 2008

This article has been downloaded from IOPscience. Please scroll down to see the full text article. 2009 Class. Quantum Grav. 26110301

(http://iopscience.iop.org/0264-9381/26/11/110301)

View the table of contents for this issue, or go to the journal homepage for more

Download details:

IP Address: 131.251.133.25

The article was downloaded on 04/04/2012 at 12:57

Please note that terms and conditions apply. 


\section{Proceedings of the 2008 Numerical Relativity Data Analysis Meeting, Syracuse University, Syracuse, NY, USA, 11-14 August 2008}

The 2008 Numerical Relativity Data Analysis (NRDA) Meeting, the second in the series, was hosted by the Department of Physics at Syracuse University, 11-14 August 2008 with 60 participants. The purpose of the NRDA meetings is to bring together two communities with a vested interest in gravitational-wave observations: the data analysis and numerical relativity communities. The first NRDA meeting was held in November 2006 at MIT. A quote of Peter Saulson's from the Matters of Gravity Newsletter puts the importance of the NRDA meetings in perspective. He wrote: 'As I sat in the back row of Rm NW14-1112 at MIT on Tuesday 7 November 2006, it suddenly struck me that we were participating in a watershed moment in the history of gravitational physics. Here, in the same room, were two communities who decades earlier had promised to help each other in a grand adventure: the detection of gravitational waves and the use of those waves to explore the frontiers of strong field gravity.' That meeting marked the first time when the two communities began to speak each other's language.

By the time of the second NRDA meeting, much progress had been made. Numerical relativists were starting to explore the binary-black-hole parameter space and were making advances in evolutions of neutron-star and neutron-star/black-hole binaries. Data analysts were investigating better algorithms for the detection of both inspiral and burst sources. Most importantly, on 14 August 2008, someone sitting in the back row of the Stolkin Auditorium in Syracuse University might have noted the beginning of real collaborations between the two communities. The meeting included presentations based on joint work by numerical relativists and data analysts. Also the participants at NRDA2008 asked tough questions about how to best use numerical relativity in gravitational wave detection, as well as showcasing some of the science that will allow us to formulate the answers to these questions.

This issue presents many of the highlights of the meeting. These include an article that summarizes the NINJA project, a collaboration between data analysts and numerical relativists that is testing data analysis pipelines on numerical relativity waveforms buried in simulated detector noise. In addition, there are several technical papers concerning the results of team efforts involved in NINJA. Also included is a review of the status of black-hole simulations, updates on black-hole and neutron-star sources of gravitational waves, accuracy tests of gravitational waveforms, binary parameter estimation methods, updates on searches using analytic and phenomenological waveforms, and a road map to the advanced LIGO detectors.

The conference organizers would like to acknowledge the financial support of the National Science Foundation under grant number PHY-0838740, and support from Syracuse University. We thank the local organizing committee of Duncan Brown, Penny Davis and Joshua Smith as well as the other members of the scientific organizing committee of Duncan Brown (Syracuse University), Sascha Husa (AEI), Badri Krishnan (AEI) and Harald Pfeiffer (CITA) for putting 
together an exciting conference. We also thank the editorial staff of the journal Classical and Quantum Gravity, especially Adam Day, Suzanne Prescott, and Joseph Tennant for their assistance, support, and patience in preparing this issue. Finally, we would like to thank the participants of NRDA2008 for making this conference so vital and energizing.

The next NRDA meeting will be held at the Albert Einstein Institute in Potsdam, Germany 6-9 July 2009. We look forward to new collaborations, and to the continued blurring of the lines between our communities as we explore the interface of numerical relativity and data analysis.

Patrick Sutton, Cardiff University

Deirdre Shoemaker, Georgia Institute of Technology

Guest Editors 